\title{
MASSES OF DB DEGENERATE DWARFS
}

\author{
Virginia Trimble
}

Astronomy Program, U. Maryland, College Park MD 20742 USA and Dept. of Physics, U. California, Irvine CA 92717 USA

Alcock (1979a,b) has suggested that DB degenerate dwarfs can only be kept observably free of accreted interstellar hydrogen if their masses are not more than about $0.3 \mathrm{M}_{\mathcal{O}}$. This is distressing because (a) photometrically determined DB radii (hence masses) are not statistically different from those of $\mathrm{DA}^{\prime}$ 's (Shipman 1979), and (b) no one quite knows how to make a degenerate dwarf with this low a mass in the age of the galaxy. Several authors (Vauclair et a1. 1979; Michaud and Fontaine 1979) have suggested that accretion is inhibited by effects of coronae and/or magnetic fields. I address here the possibility that the DB's are a somewhat higher velocity population than the general run of degenerate dwarfs. Although, as we shall see, this may imply somewhat lower than average masses for them, the dominant effect will be reduced accretion due to the high speed of the stars through the interstellar gas.

Eleven $D B^{\prime} s$ (out of a total of about $140 \mathrm{WD}$ 's) have measured radial velocities in the compilations of Greenstein and Trimble (1967), Trimble and Greenstein (1972), and Wegner (1974). The original intent in looking again at those data was to see if the gravitational redshifts could be interpreted as implying (or excluding!) significant mass differences between the DB's and other degenerate dwarfs. This doesn't work. A1though the average redshift is smaller for the DB's than for the other stars $(24$ vs $54 \mathrm{~km} / \mathrm{sec})$, the dispersion is enormous $(-69$ to $+79 \mathrm{~km} / \mathrm{sec})$, and the two for which absolute space motions are known from companions yield gravitational "redshifts" of +43 and $-3 \mathrm{~km} / \mathrm{sec}$. But another effect turned up. Among the stars with measured proper motions and (approximately!) known distances, the $\mathrm{DB}^{\prime}$ 's apparent ly have larger than average speeds relative to the Local Standard of Rest (LSR).

The precise numbers found depend on which average gravitational redshift is removed from each measured radial velocity, how the solar motion is subtracted, and how stars of measured and estimated parallaxes are weighted, but the effect always goes the same direction. For instance, weighting all stars equally, taking off the average redshift of each class separately for its members, and subtracting solar motion relative to a Population I LSR (as in Greenstein and Trimble 1967 etc。), we find for the $\mathrm{DB}^{\prime} \mathrm{s}\langle|\mathrm{U}|\rangle=47,\langle|\mathrm{~V}|\rangle=36,\langle|W|\rangle=25$, and average space motion $72 \mathrm{~km} / \mathrm{sec}$, and for all other $W^{\prime} \mathrm{s},\langle|\mathrm{U}|\rangle=38,\langle|\mathrm{v}|\rangle=$ 23, $\langle|W|\rangle=24$, and average space motion relative to the LSR of 51 $\mathrm{km} / \mathrm{sec}$. The uncertainties are about $+10 \mathrm{~km} / \mathrm{sec}$ for the $D B^{\prime} \mathrm{s}$ and +5 $\mathrm{km} / \mathrm{sec}$ for the large sample, so that the difference looks at least moderately real. 
The more simple-minded forms of accretion scale as $\mathrm{v}^{2}$, suggesting that the DB's should, on average, pick up only half as much $\left(51^{2} / 72^{2}\right)$ interstellar hydrogen as other degenerate dwarfs. This is presumably the most important effect. In addition, stellar velocity dispersions are typically age indicators, either because stars are randomly accelerated by encounters with interstellar clouds etc. over many galactic rotation periods or because galactic disc velocity structure is somewhat hierarch1cal in nature, and we sample larger and larger volumes with stellar populations of increasing age (Tinsley and Larson 1978). We might, therefore, conclude that the $\mathrm{DB}^{\prime}$ 's are somewhat older than the general run of degenerate dwarfs. Notice that this refers to age since formation from the interstellar medium, not age as a degenerate dwarf (which is determinable from surface temperature and is surely not larger for DB's than for the, typically, cooler DA's!). The greater ages for DB's combined with standard stellar evolution calculations (e.g., Paczynski 1970) imply somewhat smaller remnant masses. The relationship is difficult to make quantitative as neither velocity dispersion vs age nor initial mass vs remnant mass is well known, but if the average DA is near $0.75 \mathrm{MO}_{\mathcal{O}}$ (Trimble and Greenstein 1972), then the average DB would be about $0.6 \mathrm{M}$ (or a proportional shift from some other adopted value for the DA's).

This mass difference is presumably not large enough to affect either accretion or downward diffusion of hydrogen significantly. It may have implications for theories of degenerate dwarf formation or cooling times that will be explored later.

Acknowledgements: This work was done at the Institute of Astronomy, Cambridge, whose Director is thanked for his accustomed hospitality.

\section{REFERENCES}

Alcock, C. 1979a, Talk at California Institute of Technology

Alcock, C. 1979b, Els ewhere in this volume.

Greenstein, J.L. and Trimble, V. 1967. Astrophys. J. 149, 283.

Michaud, G. and Fontaine, G. 1979. Astrophys. J. 229, 694.

Paczyński, B. 1970. Acta Astron. 20, 47.

Shipman, H. 1979. Astrophys. J. 228, 240.

Tinsley, B.M. . and Larson, R. 1978. Astrophys. J. 221, 554.

Trimble, V, and Greenstein, J.L. 1972. Astrophys. J. 177, 441.

Vauclair, G., Vauclair, S., and Greenstein, J.L. 1979. Preprint.

Wegner, G. 1974. Mon. Not. Roy. Astron. Soc. 166, 271. 